\title{
PROPOSTA DE EXPERIMENTO SUBSTITUINDO METAL PESADO NAS AULAS DE QUÍMICA NO ENSINO SUPERIOR
}

\author{
L. C. S. SILVA ${ }^{1 *}$, M. G. L. SILVA ${ }^{2}$, F. M. MAZZÉ ${ }^{2}$ e M. C. E. S. ATAÍDE \\ ${ }^{1}$ Instituto Federal de Educação, Ciência e Tecnologia do Rio Grande de Norte \\ ${ }^{2}$ Universidade Federal do Rio Grande do Norte \\ ${ }^{3}$ Universidade Federal do Piauí \\ liviaquimili@hotmail.com*
}

Artigo submetido em janeiro/2016 e aceito em fevereiro/2016

DOI: $10.15628 /$ holos.2016.3930

\section{RESUMO}

A preocupação ambiental permeia os diferentes âmbitos de ensino e pesquisa tanto nacional como internacional. Não obstante, ainda é possível observar a presença de experimentos, em instituições de ensino superior, que provoque impacto visual como, por exemplo, a precipitação do iodeto de chumbo, um produto químico que contém metal pesado. Evitar a geração de rejeitos químicos perpassa a formação do profissional como parte do seu dia-a-dia. Este artigo relata uma proposta de substituição de um experimento nas aulas de química geral experimental do ensino superior que tem como produto o metal pesado chumbo $(\mathrm{Pb})$ envolvendo uma reação de precipitação por um outro experimento cujo precipitado não apresenta metal pesado.

PALAVRAS-CHAVE: reações de precipitação, metal pesado, rejeito químico, ensino superior.

\section{PROPOSAL FOR REPLACING EXPERIMENT HEAVY METAL IN CHEMISTRY LESSONS IN HIGHER EDUCATION}

\begin{abstract}
The environmental concern permeates different educational levels and researches both nationally and internationally. Nevertheless, it is still possible to observe the presence of experiments in higher education institutions which causes visual impact, for example, the precipitation of lead iodide, a chemical that contains heavy metal. Avoid generation of chemical waste
\end{abstract}

permeates the professional training as part of their dayto-day. This article reports a proposed replacement of an experiment in experimental general chemistry classes in higher education whose product the heavy metal lead $(\mathrm{Pb})$ involving a precipitation reaction to another with the formation of precipitate without the presence of a heavy metal.

KEYWORDS: precipitation reactions, heavy metal, chemical waste, higher education. 


\section{INTRODUÇÃO}

Nas últimas décadas, têm se dado maior ênfase ao uso de atividades experimentais ${ }^{1}$ e ao tratamento de rejeitos químicos gerados em laboratórios (Jardim, 1998; Carvalho, 1999; Cunha, 2001; Amaral et al, 2001; Afonso et al, 2003; Alberguini, Silva, Rezende, 2003, 2005; Afonso; Silveira; Oliveira; Lima, 2005; Gimenez et al, 2006; Morita, Assumpção, 2007; Machado, Mol, 2008; Giovannini, Tavares, Bendassolli, 2008; Puna, Baptista, 2008; Hirata, Filho, 2008, etc.). Entre estes estudos, há aqueles focados no gerenciamento de rejeitos químicos em instituições de ensino e pesquisa no Brasil (Giloni-Lima, Lima, 2008; Ferreira et al., 2005; Abreu, lamamoto, 2003; Bendassolli et al., 2002; Felisberto et al., 2008; Silva, Machado, 2008; Tavares; Bendassolli, 2005; Jardim, 1998). Alguns resultados apresentados por estes estudos destacam que, apesar da produção de rejeitos e resíduos na grande maioria das universidades - em especial, nos institutos e departamentos de química - ser pequena quando comparada aos processos industriais em grande escala, a gestão dos rejeitos e resíduos gerados nas atividades laboratoriais rotineiras ainda é/era insipiente devido à falta de um órgão fiscalizador, tornando o descarte inadequado uma prática ainda comum nos dias atuais.

Aliado a este aspecto, as instituições de ensino superior têm como papel formar recursos humanos, isto é, profissionais para atuarem no mercado de trabalho. A inserção de uma consciência ambiental nestes futuros profissionais é fundamental, independentemente se eles, por exemplo, atuarão ou não em uma indústria química (Giloni-Lima, Lima, 2008).

Nos cursos de formação superior, particularmente naqueles que contemplam em sua estrutura curricular o componente de química geral experimental, é fundamental que se desenvolvam habilidades operacionais e técnicas nos futuros profissionais para trabalharem em laboratórios e para compreenderem os conceitos químicos envolvidos em alguns fenômenos, correlacionando teoria e prática. Todos os pontos até aqui destacados são de interesse em nosso estudo, particularmente no que se refere à formação de químicos que possam ter uma formação que considere, em alguma medida, a preocupação com os rejeitos químicos. Neste artigo, relatamos a substituição nas aulas de química geral experimental no ensino superior de um experimento clássico de formação do precipitado de iodeto de chumbo (Mortimer, Machado, 2008), que é um produto ou rejeito químico danoso ao ambiente, por outro experimento com mesmas bases teóricas, mas cujo precipitado não apresenta metal pesado em sua composição.

\section{REJEITOS OU RESÍDUOS?}

Os termos 'rejeito e resíduo' são muito utilizados no cotidiano, mas têm significados distintos (Ataíde, 2010). Assumimos apoiados em Amaral et al. (2001, p. 421) que resíduo é "todo e qualquer material que pode ser reaproveitado, sem tratamento prévio, em algum outro experimento e rejeito

\footnotetext{
${ }^{1}$ A literatura apresenta uma diversidade de termos tais como práticas de laboratório, experiências, aulas práticas entre outras, mas assumiremos o termo atividades experimentais.
} 
seria todo e qualquer resíduo que não apresenta utilidade alguma, pelo menos até o momento, e que, portanto, precisa ser descartado".

No contexto das instituições de ensino superior, tanto no âmbito do funcionamento dos laboratórios de ensino como de pesquisa, não basta apenas fazer a diferenciação entre resíduo e rejeito, mas também reconhecer aqueles que trazem risco à saúde e ao meio ambiente e propor alternativas para minimizar ou eliminar os riscos.

Nos últimos anos, a preocupação com o ambiente e sua preservação para as gerações futuras vem crescendo e se incorporando no cotidiano das pessoas, nos currículos dos cursos superiores, nas legislações nacionais, entre outros. Uma alternativa que vem sendo empregada para a geração e descarte de resíduos químicos que trazem prejuízos ao meio ambiente e a sociedade é a reciclagem ou diminuição/controle (Alberguini; Silva; Rezende, 2005), particularmente para os resíduos sólidos.

A legislação brasileira classifica os resíduos sólidos em perigosos e não perigosos. Os não perigosos são subdivididos em inerte e não inerte. Esta norma define que os resíduos sólidos são aqueles gerados a partir de atividades que tornem inviável o descarte na rede pública de esgoto ou corpos de água (Brasil, 2011). Até algum tempo atrás, era uma prática comum realizar o descarte no ambiente após a diluição, pois havia a visão ingênua de que tal técnica não ofereceria riscos (Felisberto et al., 2008). Particularmente, a legislação brasileira chama a atenção para os metais pesados, seus compostos e para os produtos orgânicos sintéticos não biodegradáveis, os quais mesmo após passarem pelo processo de diluição, ainda podem trazer prejuízos aos mananciais aquíferos (lençóis freáticos) em função da tendência de serem absorvidos pelo meio ambiente, concentrando-se nos organismos vivos e podendo ser letal.

Dentre as Diretrizes do Plano Nacional referentes aos resíduos sólidos, inclui-se a "educação para o consumo sustentável" que tem como objetivo despertar no indivíduo a reflexão sobre suas ações para com o ambiente. Neste sentido, as instituições de ensino superior têm direto envolvimento e responsabilidade (Brasil, 2011).

\subsection{O papel das instituições de ensino superior como geradoras de REJEITOS químicos}

Ao falar de produção de rejeitos químicos, em geral, é comum pensar que o locus da geração é na indústria ou no laboratório (este, por sua vez, pode ser de uma universidade, instituto ou centro de pesquisa, entre outros).

As instituições de ensino superior têm papel relevante nas discussões sobre a questão dos rejeitos químicos, não somente pelo fato de comporem o grupo de produtores de rejeitos químicos, mesmo que em menor quantidade se comparado às indústrias, mas também pelo seu papel enquanto formadores de recursos humanos (Amaral et al., 2001; Ataíde, 2010; Abreu; lamamoto, 2003).

A responsabilidade de quem gera rejeitos deve considerar ações como: prevenir a geração de qualquer tipo de rejeito, seja ele perigoso ou não; diminuir a quantidade de rejeitos gerados que sejam perigosos; segregar e concentrar rejeitos de forma viável e economicamente possível; reusar 
ou reciclar os rejeitos; realizar tratamento ou manter o rejeito de modo que possa ser tratado (Ataíde, 2010).

Jardim (1998) destaca que o papel das universidades perpassa pela discussão sobre a questão ambiental como, por exemplo, pela proposição de um plano de gestão para o gerenciamento dos resíduos e rejeitos gerados contemplando o ativo e o passivo. $O$ ativo é gerado em atividades de rotina dentro da própria universidade e o passivo é estocado sem caracterização. Os programas de gerenciamento requerem quatro condições básicas, a saber: o apoio da instituição; a priorização do ser humano; a divulgação das metas previstas e a reavaliação contínua dos resultados. A ausência de tratamento ou de destinação pode comprometer o solo, o ar e os recursos hídricos, além de criar um habitus inconsciente de descarte inadequado. Além disso, deve-se considerar que o gerenciamento depende de vários fatores, tais como o tipo de resíduo e de rejeito, a forma de geração, o acondicionamento na fonte geradora, a coleta, o transporte, o processamento, a recuperação e a destinação final (Alberguini; Silva; Rezende, 2005).

Ao considerar estes pontos, muitas instituições de ensino superior brasileiras desenvolvem planos de gerenciamento levando em conta suas particularidades. Dentre as ações mais usuais, destacam-se: a criação de programas de reutilização e descarte de rejeitos produzidos nas aulas de laboratório incluindo em, alguns casos, a incineração na própria instituição ou em órgãos e empresas credenciadas; a criação de banco de dados com cadastramento de insumos, resíduos e rejeitos e sua disponibilização online para possível reutilização; a criação de projetos de tratamento de resíduos sólidos nos cursos de ensino superior como parte da formação dos futuros profissionais; a implementação de disciplina na estrutura curricular do curso de ensino superior incluindo visitas a empresas, seminários, palestras; a criação de comissão interna de segurança para elaboração de manual e práticas de segurança relacionadas ao gerenciamento e tratamento de rejeitos e resíduos produzidos na instituição; a criação de programas de caracterização e análise do passivo, entre outras (Ferro, Longhinotti, Borges, 2007; Amaral et al., 2001; Cunha, 2001; Alberguini, Silva, Rezende, 2003; Afonso et al., 2003; Pugliesi et al., 2007; Afonso, Silveira, Oliveira, 2005). Assim, várias instituições do país vêm desenvolvendo mecanismos e ações para reduzir e gerenciar os rejeitos produzidos pelas suas atividades que, por sua vez, devem acompanhar o aspecto formativo dos profissionais (Ataíde, 2010).

\subsection{Ações desenvolvidas nos cursos de química da ufrn}

Diferentes unidades acadêmicas da UFRN geram, segundo relatório técnico da Divisão de Meio Ambiente, aproximadamente 5 toneladas de rejeitos por ano (UFRN, 2009). Dentre as ações desenvolvidas no Instituto de Química, podem ser destacadas: a criação do banco de reagentes (online); central analítica; comissão interna; separação e coleta de resíduos e rejeitos com posterior incineração em empresa credenciada; estudos de substituição de experimentos.

Neste contexto, algumas práticas experimentais de grande impacto visual, consolidadas nas disciplinas e que, muitas vezes, estão presentes em livros do ensino superior (Atkins, 2011) como a observada na Figura 1 para a precipitação do iodeto de chumbo, devem ser substituídas por outras práticas experimentais que também apresentem boa visualização e abordem o mesmo conteúdo, mas que não formem rejeitos (Ataíde, Silva e Dantas, 2009). 


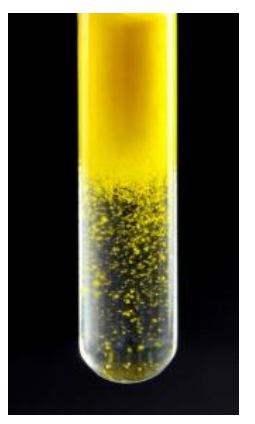

Figura 1: Reação de precipitação do iodeto de chumbo

Fonte: http://imagens.tabelaperiodica.org/wp-content/uploads/2013/07/iodeto-chumbo-pptado.jpg

A equação química que representa a reação química de precipitação de iodeto de chumbo pode ser escrita como:

$$
\mathrm{Pb}\left(\mathrm{NO}_{3}\right)_{2}(\mathrm{aq})+2 \mathrm{KI}(\mathrm{aq}) \rightarrow 2 \mathrm{KNO}_{3}(\mathrm{aq})+\mathrm{Pbl}_{2}(\mathrm{~s})
$$

Ao observar o contexto acadêmico, pode-se dizer que alguns experimentos, como o exemplo citado na equação 1 , continuam a gerar rejeitos ainda que em pequena escala e que, com o passar dos anos, acumulam-se, exigindo um olhar mais cuidadoso para seu tratamento.

\section{ASPECTOS METODOLÓGICOS}

\subsection{CONTEXTO DAS MUDANÇAS}

Em 2010, ocorreram mudanças na estrutura curricular dos cursos de Licenciatura, Bacharelado e de Química do Petróleo da UFRN. Estas mudanças promoveram a revisão de componentes curriculares, de atividades experimentais, de atividades de extensão, de programas de formação complementar, fornecendo uma nova perspectiva para o Projeto Político do Curso visando a atender as Diretrizes Curriculares Nacionais e a demanda do mercado de trabalho.

Neste contexto, o componente curricular Química Geral Experimental foi totalmente reestruturado para atender a especificidade formativa de cada curso, tendo como foco o perfil profissional e reconhecendo a relevância dos conceitos químicos envolvidos no âmbito do conteúdo programático. Ao longo desta reestruturação, todas as atividades experimentais que geravam rejeitos foram substituídas e adequadas à realidade acadêmica.

Uma das mudanças nas práticas de laboratório se refere à substituição da reação química de formação de sal de metal pesado (precipitado amarelo característico do iodeto de chumbo apresentada na equação 1) por outro sal (um precipitado branco de carbonato de cálcio, equação 2) sem toxicidade (Machado; Mol, 2008). Este experimento foi inicialmente proposto para o curso de Licenciatura em Química no componente Ensino de Química I e, posteriormente, incorporado na disciplina de Química Geral Experimental oferecida aos cursos de Bacharelado em Química e Bacharelado em Química do Petróleo, bem como para todos os outros cursos de graduação da UFRN que contemplam tal componente em sua estrutura curricular (por exemplo, as engenharias, física, entre outros). 


\subsection{O experimento: DISCUTINDO RESULTADOS E aspectos didáticos e técnicos}

O experimento proposto é simples, utiliza materiais com custos relativamente baixos, é de domínio público (Mortimer, Machado, 2008; Silva, Machado, 2008) e teve sua discussão apresentada de forma mais aprofundada para os graduandos no ensino superior.

Do ponto de vista técnico e operacional para a realização deste experimento no laboratório de química, são necessárias as seguintes vidrarias e soluções:

- Cinco tubos de ensaio limpos e secos incluindo o suporte para tubos;

- Solução de carbonato de sódio a 0,5 mol..-1;

- Solução de cloreto de cálcio 0,5 mol. $\mathrm{L}^{-1}$;

É importante ressaltar que os tubos de ensaio devem ser identificados porque serão colocados volumes diferentes dos reagentes, conforme a seguir:

Tabela 1: Quantidades das soluções utilizadas no experimento

\begin{tabular}{c|c|c}
\hline Tubo & $\begin{array}{c}\text { Volume em mL de } \\
\text { sol. } \mathrm{Na}_{2} \mathrm{CO}_{3} \text { à } 0,5 \mathrm{~mol} . \mathrm{L}^{-1}\end{array}$ & $\begin{array}{c}\text { Volume em } \mathrm{mL} \mathrm{de} \\
\text { sol. } \mathrm{CaCl}_{2} \text { à } 0,5 \mathrm{~mol} . \mathrm{L}^{-1}\end{array}$ \\
\hline $\mathrm{A}$ & 10 & 2 \\
\hline $\mathrm{B}$ & 8 & 4 \\
\hline $\mathrm{C}$ & 6 & 6 \\
\hline $\mathrm{D}$ & 4 & 8 \\
\hline $\mathrm{E}$ & 2 & 10 \\
\hline
\end{tabular}

Uma sugestão de abordagem metodológica é indagar aos graduandos em qual dos tubos ocorrerá a formação de maior quantidade de precipitado e se os produtos da reação provocarão danos ao ambiente. Para responderem estes questionamentos, os graduandos deverão fazer a previsão teórica dos cálculos que implica em representar a equação química balanceada com os respectivos produtos formados, identificar o reagente limitante, analisar a formação de produto em função das tabelas de solubilidade, além de interpretar quimicamente os reagentes e produtos com relação à segurança ambiental.

A comparação dos resultados teóricos com os experimentais pode promover o desenvolvimento de habilidades operacionais como o controle de variáveis e o manuseio de diferentes equipamentos.

Após realizada a prática experimental, é possível, ainda, sugerir aos graduandos que determinem o rendimento da reação química ocorrida ou que proponham experimentos, sem um roteiro prévio, para que este rendimento possa ser calculado. Esta proposta de atividade pode favorecer o desenvolvimento de habilidades do pensamento de alta ordem. Uma possibilidade para solucionar esta questão inclui a deposição do precipitado formado com o auxílio de uma centrífuga ou aguardar o repouso do precipitado no fundo de tubo de ensaio (decantação), separar o líquido sobrenadante e pesar o produto formado. A figura 2 a seguir apresenta o resultado das reações químicas nos tubos de ensaio: 


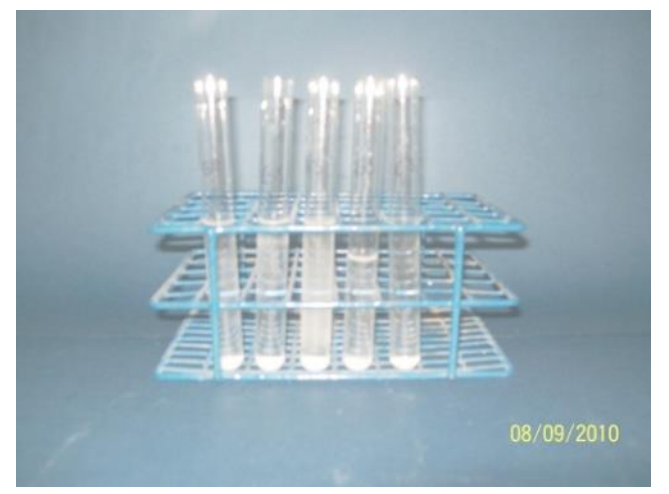

Figura 2: Reações químicas de carbonato de sódio e cloreto de cálcio em volumes diferentes Fonte: Autoria própria

No caso da reação química ocorrida nos tubos de ensaio, ao escrever a equação geral obtemos a equação (2):

$$
\mathrm{Na}_{2} \mathrm{CO}_{3}(\mathrm{aq})+\mathrm{CaCl}_{2}(\mathrm{aq}) \rightarrow 2 \mathrm{NaCl}(\mathrm{aq})+\mathrm{CaCO}_{3}(\mathrm{~s})
$$

Como os carbonatos são insolúveis, exceto aqueles que contem íon de metal alcalino ou íon amônio, a representação da equação iônica simplificada para esta reação é dada pela equação (3) a seguir:

$$
\mathrm{Ca}^{2+}(\mathrm{aq})+\mathrm{CO}_{3}{ }^{2-}(\mathrm{aq}) \rightarrow \mathrm{CaCO}_{3}(\mathrm{~s})
$$

A constante de equilíbrio para um equilíbrio de solubilidade entre um sólido e sua forma dissolvida é dada pelo produto de solubilidade ou Kps. Deste modo, no equilíbrio temos a equação (4) com o respectivo Kps:

$$
\begin{aligned}
& \mathrm{CaCO}_{3}(\mathrm{~s}) \leftrightharpoons \mathrm{Ca}^{2+}(\mathrm{aq})+\mathrm{CO}_{3}{ }^{2-}(\mathrm{aq}) \\
& \mathrm{Kps}=\left[\mathrm{Ca}^{2+}\right]\left[\mathrm{CO}_{3}{ }^{2-}\right] \\
& \mathrm{Kps}=8,7 \times 10^{-9} \text { a } 25^{\circ} \mathrm{C}
\end{aligned}
$$

A precipitação ocorre quando o quociente da reação (Qps) é igual ou maior que o produto de solubilidade (Kps). Assim, se Qps > Kps para equação 4, a reação tenderá a produzir o $\mathrm{CaCO}_{3}(\mathrm{~s})$ até que o equilíbrio químico seja atingido. $\mathrm{O} \mathrm{Kps}$ pode ser usado como qualquer outra constante de equilíbrio, mas como interações íon-íon em eletrólitos concentrados podem ter interpretação complicada, o Kps é geralmente significativo apenas para sais poucos solúveis.

Nesta atividade prática proposta, podemos destacar que, com relação aos aspectos didáticopedagógicos, a execução e discussão do experimento de formação do precipitado de carbonato de cálcio (equação 2) permitem o desenvolvimento de habilidades procedimentais característicos da química, além de habilidades cognitivo-linguísticas como observar, prever e explicar as reações químicas envolvidas. Permite, dependendo do planejamento do professor, o desenvolvimento de habilidades de pensamento de alta ordem, ao propiciar situações em que o graduando possa investigar e apresentar argumentos plausíveis para os resultados observados, ou seja, não é somente a reprodução ou comprovação de um dado conceito químico, mas pode constituir uma ferramenta importante para o desenvolvimento de habilidades profissionais. Além disso, o experimento proposto trabalha com materiais cujos resíduos não agridem o meio ambiente e, deste 
modo, questões ambientais também podem ser abordadas em sala de aula. Como sugestão, o resíduo de carbonato de cálcio formado poderia, ainda, ser reutilizado em uma prática posterior como, por exemplo, em uma reação de neutralização com um ácido.

\section{CONSIDERAÇÕES FINAIS}

O uso de experimentos de forma didática, a partir de uma mudança metodológica, vai além da sua apresentação por meio de um roteiro que confirme um dado conceito científico, ou seja, as práticas experimentais devem visar ao desenvolvimento de habilidades cognitivo-linguísticas como observar, prever e explicar os fenômenos químicos e, assim, contribuir para a aproximação entre a teoria e a prática.

Ao abordar um experimento que substitua a geração de rejeitos químicos, problematizando com os graduandos, pode-se proporcionar outros elementos formativos, como a preocupação com o meio ambiente e a saúde dos seres humanos. Além dos aspectos químicos, é importante que o docente problematize o descarte dos produtos gerados na reação química, alertando-os para as questões ambientais, para o entendimento generalista e equivocado de que após a diluição de qualquer rejeito é possível descartá-lo na rede de esgoto e para a crença de que uma pequena produção de rejeito não causará danos ao ambiente e as pessoas. Debater tais visões distorcidas é fundamental para a formação de um profissional consciente do seu papel na sociedade.

Entendemos que uma discussão aprofundada do experimento pode abordar, além das questões ambientais, conteúdos como reações químicas, representação de uma reação química, cálculos estequiométricos para determinação das quantidades de produtos gerados, rendimento da reação, identificação do reagente limitante, desenvolvimento de habilidades operacionais e controle de variáveis.

\section{REFERÊNCIAS BIBLIOGRÁFICAS}

1. ABREU, D.G.; IAMAMOTO, Y. Relato de uma experiência pedagógica no ensino de química: formação profissional com responsabilidade ambiental. Química Nova, v. 26, n. 4, p. 582-584, 2003.

2. AFONSO, J.C. et al. Gerenciamento de resíduos laboratoriais: recuperação de elementos e preparo para descarte final. Química Nova, v. 26, n. 4, p. 602-611, 2003.

3. AFONSO, J. C.; SILVEIRA, J. A.; OLIVEIRA, A.S.; LIMA, R.M.G. Análise sistemática de reagentes e resíduos sem identificação. Química Nova, v. 28, n. 1, p. 157-165, 2005.

4. ALBERGUINI, L.B.A.; SILVA, L.C.; REZENDE, M.O.O. Tratamento de resíduos químicos: Guia prático para a solução dos resíduos químicos em instituições de ensino superior. São Carlos: Ed. RiMa, 2005.

5. _ Laboratório de resíduos químicos do Campus USP - São Carlos: Resultados da experiência pioneira em gestão e gerenciamento de resíduos químicos em campus universitários. Química Nova, v. 26, n. 2, p. 291-295, 2003.

6. AMARAL, S.T. et al. Relato de uma experiência: recuperação e cadastramento de resíduos dos 
laboratórios de graduação do Instituto de Química da Universidade Federal do Rio Grande do Sul. Química Nova, v. 24, n. 3, p. 419-423, 2001.

7. ATAÍDE, M.C.S. Experimentos que geram rejeitos químicos com metais pesados em escolas da educação básica. Natal, 2010. Dissertação de mestrado. Pós-Graduação em Ensino de Ciências Naturais e Matemática, Universidade Federal do Rio Grande do Norte, 2010.

8. ATAÍDE, M.C.E.S.; SILVA, M.G.L.; DANTAS, J.M. Experimentos em livros didáticos: aspectos relacionados a segurança e rejeitos químicos. Experiências em Ensino de Ciências, v. 4, n. 3, p. 61-78, 2009.

9. $\quad$ ATKINS, P.; JONES, L. Princípios da Química. 5ạ Ed. Bookman Ed. 2011.

10. BRASIL. Plano Nacional de Resíduos Sólidos. Ministério do Meio Ambiente. Brasília, 2011. Disponível em <http://www.mma.gov.br>.

11. BENDASSOLLI, J. A. et al. Reciclagem de cobre proveniente de analisador automático de carbono e nitrogênio. Química Nova, v. 25, n. 2, p. 312-315, 2002.

12. CARVALHO, P. R. Boas práticas químicas em biossegurança. Rio de Janeiro: Interciência, 1999.

13. CUNHA, C.J. O programa de gerenciamento dos resíduos laboratoriais do depto de química da UFPR. Química Nova, v. 24, n.3, p.424-427, 2001.

14. FELISBERTO, R. et al. De resíduo a insumo: a construção do caminho para uma química mais limpa através de um projeto de ensino. Química Nova, v. 31, n. 1, p. 174-177, 2008.

15. FERREIRA et al. Gerenciamento de resíduos químicos em instituições de ensino e pesquisa. Química Nova, v. 28, n. 1, p. 3, 2005.

16. FERRO, E. S.; LONGHINOTTI, E.; BORGES, S. S. S. Reaproveitamento de soluções utilizadas em laboratórios de Química Analítica. In: Congresso Brasileiro de Química, 47. 2007, Natal. Anais... Natal: CBQ, 2007.

17. GIOVANNINI, J.G.; TAVARES, G.A.; BENDASSOLLI, J.A. Avaliação das técnicas de precipitação química e encapsulamento no tratamento e destinação conjunta de resíduos líquidos contendo cromo e vidrarias de laboratório. Química Nova, v. 31, n. 3, p. 676-679, 2008.

18. GILONI-LIMA, P. C.; LIMA, V. A. Gestão integrada de resíduos químicos em instituições de ensino superior. Química Nova, v. 31, n. 6, p. 1595-1598, 2008.

19. GIMENEZ, S.M.N. et al. Diagnóstico das condições de laboratórios, execução de atividades práticas e resíduos químicos produzidos nas escolas de ensino médio de Londrina-PR. Química Nova na Escola, n. 23, maio, p. 32-36, 2006.

20. GONÇALVES, M. et al. Gerenciamento de resíduos sólidos na Universidade Tecnológica Federal do Paraná Campus Francisco Beltrão. Revista Brasileira de Ciências Ambientais, v. 15, março, p. 79-84, 2010.

21. HIRATA, M. H.; FILHO, J. M. Manual de biossegurança. São Paulo: Manole, 2008.

22. JARDIM, W. F. Gerenciamento de resíduos químicos em laboratórios de ensino e pesquisa. Química Nova, v. 21, n.5, p. 671-673, 1998.

23. MACHADO, P. L.; MOL, G. S. Experimentando com Segurança. Química Nova na Escola, v. 27, n. 1, p. 57-60. 2008. 
24. MORITA, T.; ASSUMPÇÃO, R.M.V. Manual de soluções, reagentes e solventes - padronização, preparação, purificação, indicadores de segurança e descarte de produtos químicos. São Paulo: Ed. Blucher, 2007.

25. MORTIMER, E.F.; MACHADO, A.H. Química. 1ạ. Ed. Scipione. São Paulo, 2007.

26. SILVA, R. R.; MACHADO, P. F. L. Experimentação no ensino médio de química: a necessária busca da consciência ético-ambiental no uso e descarte de produtos químicos - um estudo de caso. Ciência \& Educação, v. 14, n. 2, p. 233-249, 2008.

27. PENATTI, F. E.; GUIMARÃES, S. T. L. Avaliação dos riscos e problemas ambientais causados pela disposição incorreta de resíduos de laboratórios. Geografia Ensino \& Pesquisa, v. 15, n. 1, jan./abr., p. 43-52, 2011.

28. PUNA, J.F.B.; BAPTISTA, B.S. A gestão integrada de resíduos sólidos urbanos - Perspectiva ambiental e econômico-energética. Química Nova, v. 31, n.3, p. 645-654, 1998.

29. PUGLIESI et al. Gestão de resíduos no IQ-UFF - Parte III. In: CONGRESSO BRASILEIRO DE QUÍMICA, 47. 2007, Natal. Anais... Natal: CBQ, 2007.

30. TAVARES, G. A.; BENDASSOLLI, J. A. Implantação de um programa de gerenciamento de resíduos químicos e águas servidas nos laboratórios de ensino e pesquisa no CENA/USP. Química Nova, v. 28, n. 4, p. 732-738, 2005.

31. UFRN. Relatório Interno - Divisão de Meio Ambiente/UFRN, 2009 (material mimeo). 